\title{
Measurement of entangled states via atomic beam deflection in Bragg's regime
}

\author{
Ashfaq H. Khosa, ${ }^{1}$ Manzoor Ikram, ${ }^{1,2}$ and M. Suhail Zubairy ${ }^{1,3}$ \\ ${ }^{1}$ Department of Electronics, Quaid-i-Azam University, Islamabad, Pakistan \\ ${ }^{2}$ Applied Physics Division, Pakistan Institute of Nuclear Science and Technology, P. O. Nilore, Islamabad, Pakistan \\ ${ }^{3}$ Department of Physics, Texas A\&M University, College Station, Texas, 77843, USA
}

(Received 14 July 2003; revised manuscript received 15 October 2003; published 11 November 2004)

\begin{abstract}
We propose a scheme for the measurement of joint photon statistics and Wigner function of the entangled field states between two separate cavities. The scheme utilizes the momentum state of the two-level atoms in Bragg's regime. The probability of finding the atom in any one of the momentum states is the product of joint photon statistics and an oscillatory function. The argument of the function contains the information of the joint photon numbers in two cavities. We use the method of state reduction to a single set of joint photon numbers, which is a consequence of the measuring sequence. The repeated measurement of the set of photon numbers gives the joint photon statistics of the entangled field state. The complete entangled state can be reconstructed as a Wigner function from the knowledge of photon statistics of the coherently displaced entangled state.
\end{abstract}

DOI: 10.1103/PhysRevA.70.052312 PACS number(s): 03.67.-a, 03.65.Ta, 42.50.Ar, 03.65.Ud

\section{INTRODUCTION}

The entangled state lies at the base of several interesting phenomena related to the newly emerging field of quantum informatics [1-5]. It is therefore of interest to find schemes for the measurement of the entangled states in various systems. In this paper, we consider a method based on atom optics to measure an arbitrary entangled state in a cavity QED system.

Generally, the quantum state in a single experiment cannot be measured precisely. One needs to perform many experiments on identically prepared systems to infer the quantum state from the recorded statistical distributions. Faridani et al. [6] have experimentally verified this idea in a quantum optical system proposed by Vogel and Risken [7]. In this scheme, a homodyne detection method is employed to measure the quadrature distributions of identically prepared light pulses. The Wigner function is then reconstructed from the measured quadrature distributions. The other methods for the measurement of the cavity field are based on dispersive atom-field coupling in a Ramsey method of separated oscillatory fields [8], absorption and emission spectrum [9], and atomic beam deflection [10-12]. These schemes are concentrated over the measurement of a single mode field in the high- $Q$ cavity.

Among these schemes the atomic scattering method provides a good tool for the measurement of field photon statistics, as the momentum distribution of the deflected atoms is a function of field photon numbers [10-15]. The atomic diffraction from the electromagnetic field may be divided into two regimes, one in which the recoil energy of the field is much greater than the Rabi frequency (Bragg's regime $[11,13,16])$ and the other in which the recoil energy is much less than the Rabi frequency (Raman-Nath regime $[10,12]$ ). Bernhardt and Shore [13] gave the theory of Bragg diffraction of atomic de Broglie waves from the electromagnetic fields. Since then, there have been many papers; for example, those that report Bragg's diffraction of the matter wave from the optical fields. These experiments demonstrate up to eighth order of Bragg's diffraction [17,18]. All these experi- ments are performed with the classical field. However, the advancement in technology has made it possible to realize the diffraction of atoms from quantized cavity field [19].

We deal with the utilization of atomic diffraction in the Bragg regime for the measurement of joint photon statistics of the entangled field state in two separate cavities. There are a few schemes reported for the reconstruction of the multimode field [20-24]. Kim and Agarwal [21] used the idea that the probability of atomic inversion after a two-level atom interacts with a cavity field which is directly related to the Wigner characteristic function [25]. The scheme by Davidovich et al. uses the Ramsey-type setup to reconstruct the quantum state [24]. Earlier we proposed another scheme based on Autler-Townes spectroscopy for the reconstruction of the two-mode entangled state in a high- $Q$ cavity [23].

In this paper we show that the entangled field state in spatially separated cavities can be measured using the techniques of Bragg's diffraction of two-level atoms from the field. We consider the deflection of atoms from far detuned high- $Q$ cavities in a first-order Bragg regime and develop a measurement scheme for the entangled field state. The method adopted here is the reduction of the entangled field state to a state having a set of $m_{A}$ and $m_{B}$ photons in cavities $A$ and $B$, respectively, and then measuring it by a quantum nondemolition method. Repeated measurements of such a set of joint photon numbers give us the information about the diagonal density matrix elements of entangled field state and hence the joint photon statistics in two separate cavities. To obtain the information about the off-diagonal matrix elements of the field, we propose to reconstruct the Wigner function of the entangled field state. For this purpose, we propose displacing the field state in two cavities by injecting coherent states in each cavity [26-28]. The displaced photon statistics can be used to reconstruct the Wigner function of entangled field state in separate cavities in a straightforward manner.

\section{MEASUREMENT OF JOINT PHOTON STATISTICS}

We consider a two-mode entangled field state present in two separate high- $Q$ cavities. The state vector of the en- 


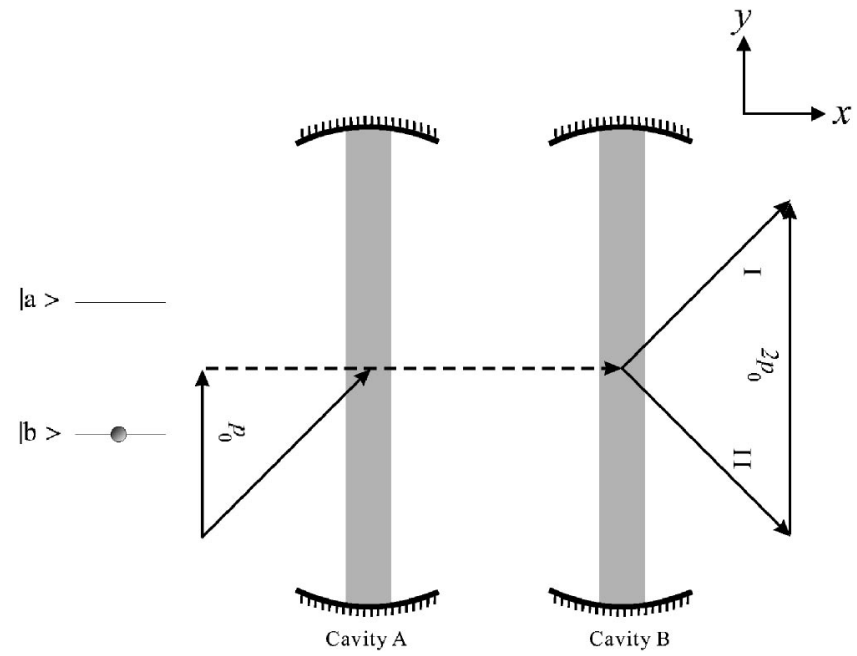

FIG. 1. Schematics of a two-level atom interacting with a standing wave entangled field in two separate cavities. Here the atom has well defined momentum state $\left|p_{0}\right\rangle$ along the wave propagation direction ( $x$ axis). After the interaction with the standing wave field of entangled cavities, the atom either retains the state of the momentum $\left|p_{0}\right\rangle$ or reverses the direction of longitudinal momentum component $\left|p_{-2}\right\rangle$.

tangled field can be written as $\left|\Psi_{A B}\right\rangle=\Sigma_{m_{A}, m_{B}} C_{m_{A}, m_{B}}\left|m_{A}, m_{B}\right\rangle$, where $C_{m_{A}, m_{B}}$ is the joint probability amplitude of having $m_{A}$ photons in cavity $A$ and $m_{B}$ photons in cavity $B$. There are many schemes reported for the preparation of entangled field states [29]. We present a scheme for the measurement of such kind of entangled states by considering a generalized entangled field state as mentioned above. The schematic of our scheme is shown in Fig. 1, in which a two-level atom interacts off-resonantly with the standing wave fields of the cavities in cascade fashion. We take the atoms having detuning $\Delta_{i}=\nu_{i}-w_{0}$, between the atomic transition frequency $w_{0}$ and the frequency of the cavity mode $\nu_{i}$, where the subscript $i=A$ or $B$ is related to the field mode present in cavity $A$ or $B$, respectively. The atom first interacts with the field present in the cavity $A$. It then interacts, on its way, with the second cavity $B$. The probe in this scheme is the momentum states of the atoms in Bragg regime. In this regime, it is assumed, that the momentum component of the atoms along transverse direction is very large so it can be treated classically. At the same time, the atoms have well-defined momentum state in the direction of wave propagation of the field, i.e., the longitudinal component of the atomic momentum is well defined, and we can treat it quantum mechanically. The atoms are prepared in the ground state $|b\rangle$, with initial momentum state $\left|p_{0}\right\rangle$ in the direction of wave propagation. After interacting with two cavities the atoms are detected in either of the two momentum states, i.e., the deflected or undeflected. We suggest keeping the injection rate of the atoms very low so that there is only one atom interacting with the cavity mode at a time.

If $g_{i}$ is the coupling constant of the atom-field interaction and $a_{i}\left(a_{i}^{\dagger}\right)$ is annihilation(creation) operator of the field mode in the two cavities, then the atom-field interaction Hamiltonian (in cavity $i$ with $i=A$ or $B$ ) in the interaction picture under dipole and rotating wave approximation can be written as

$$
H_{i}=\frac{\hat{\mathbf{P}}_{x}^{2}}{2 M}+\hbar \frac{\Delta_{i}}{2} \sigma_{z}+\hbar g_{i} \cos (\mathbf{k} \cdot \hat{\mathbf{x}})\left[\sigma_{+} a_{i}+\sigma_{-} a_{i}^{\dagger}\right]
$$

where $M$ is the mass of the atom, $\sigma_{+}\left(\sigma_{-}\right)$is the atomic raising(lowering) operator, $\sigma_{z}$ is the atomic inversion operator, $\hat{\mathbf{x}}$ and $\hat{\mathbf{P}}_{x}$ are the position and momentum operators for the center-of-mass motion of the atom along $x$ axis, respectively. Here we neglect the constant momentum components along the transverse direction as discussed earlier.

We first specialize to the atom-field interaction in the cavity $A$. The wave function of the system at an arbitrary time in the discrete momentum space can be written as

$$
\begin{aligned}
\left|\Psi\left(t_{A}\right)\right\rangle= & \exp \left[-i \frac{p_{o}^{2} t_{A}}{2 M \hbar}\right] \sum_{l=-\infty}^{\infty} \sum_{m_{A}, m_{B}}\left[e^{-i \Delta_{A} t_{A} / 2} c_{m_{A}-1, m_{B}}^{a, p_{l}}\left(t_{A}\right)\right. \\
& \left.\times\left|a, m_{A}, m_{B}, p_{l}\right\rangle+e^{-i \Delta_{A} t_{A} / 2} c_{m_{A}, m_{B}}^{b, p_{l}}\left(t_{A}\right)\left|b, m_{A}, m_{B}, p_{l}\right\rangle\right],
\end{aligned}
$$

where $c_{m_{A}, m_{B}}^{j, p_{l}}\left(t_{A}\right)$ is the probability amplitude indicating the atom in internal state $j=a$ or $b$ after interaction time $t_{A}$, exiting with momentum $\mathbf{p}_{l}$ after $l$ interactions. The summation over $l$ is due to the cumulative nature of the atomic momentum that keeps on increasing or decreasing depending upon its initial value to conserve the energy of the system. Here the phase factor is introduced for the sake of simplicity.

The Schrödinger equation leads to a set of general infinite coupled differential equations of different atomic momenta. We assume that the atom has a large detuning as compared to the recoil frequency (i.e., $\Delta_{i} \gg \hbar k_{i}^{2} / 2 M$ ). Under this condition we can ignore the recoil frequency in comparison with the detuning [11]. Here it is clear that the above limit also prevents the decoherence effects due to spontaneous emission.

In Bragg's regime the atom after the interaction with the field may either go undeflected or it may get deflected. The angle of the deflection depends upon the number of interactions with the cavity field. For dispersive atom-field interaction the atom goes through complete cycles of consecutive excitations and deexcitations. Therefore, the atom emerges from the cavity in the same initial ground state conserving energy and with a total momentum change of $l \hbar k_{i}$ in the direction of wave propagation (i.e., for each complete cycle the momentum transferred to the atom is zero or $\left.2 \hbar k_{i}[13]\right)$. Also, for the diffraction of the atomic matter wave in Bragg's regime the energy associated with the recoil frequency must be much larger than the energy associated with effective Rabi frequency, i.e., $\hbar k_{i}^{2} / 2 M \gg g_{i}^{2} m_{i} / 4 \Delta_{i}$ (in case of offresonant atom-field interaction). In the set of differential equations obtained by the Schrödinger equation, we apply the above approximations and obtain a closed set of differential equations for the probability amplitudes $c_{m_{A}, m_{B}}^{b, p_{0}}\left(t_{A}\right)$ and $c_{m_{A}, m_{B}}^{b, p_{-}}\left(t_{A}\right)$. Moreover, following the approach given in Ref. [16], we adiabatically eliminate the fast varying probability amplitudes. By concentrating on the first-order Bragg's diffraction we reach at the general solution of the nonvanishing 
probability amplitudes $c_{m_{A}, m_{B}}^{b, p_{0}}\left(t_{A}\right)$ and $c_{m_{A}, m_{B}}^{b, p_{2}}\left(t_{A}\right)$ given by

$$
\begin{aligned}
c_{m_{A}, m_{B}}^{b, p_{0}}\left(t_{A}\right)= & \exp \left[i \frac{g_{A}^{2} m_{A}}{2 \Delta_{A}} t_{A}\right]\left[c_{m_{A}, m_{B}}^{b, p_{0}}(0) \cos \left(\frac{g_{A}^{2} m_{A}}{4 \Delta_{A}} t_{A}\right)\right. \\
& \left.+i c_{m_{A}, m_{B}}^{b, p_{-2}}(0) \sin \left(\frac{g_{A}^{2} m_{A}}{4 \Delta_{A}} t_{A}\right)\right], \\
c_{m_{A}, m_{B}}^{b, p_{-2}}\left(t_{A}\right)= & \exp \left[i \frac{g_{A}^{2} m_{A}}{2 \Delta_{A}} t_{A}\right]\left[c_{m_{A}, m_{B}}^{b, p_{-2}}(0) \cos \left(\frac{g_{A}^{2} m_{A}}{4 \Delta_{A}} t_{A}\right)\right. \\
& \left.+i c_{m_{A}, m_{B}}^{b, p_{0}}(0) \sin \left(\frac{g_{A}^{2} m_{A}}{4 \Delta_{A}} t_{A}\right)\right] .
\end{aligned}
$$

The initial conditions of the system as discussed previously are that the atoms are in ground state $|b\rangle$ with longitudinal momentum $\left|p_{0}\right\rangle$, we can write the same as $c_{m_{A}, m_{B}}^{b, p_{0}}(0)$ $=c_{m_{A}, m_{B}}$ and $c_{m_{A}, m_{B}}^{b, p_{2}}(0)=0$, where $c_{m_{A}, m_{B}}$ is the initial probability amplitudes of the entangled state present in the two separate cavities. Substituting these initial conditions give

$$
\begin{gathered}
c_{m_{A}, m_{B}}^{b, p_{0}}\left(t_{A}\right)=c_{m_{A}, m_{B}} \exp \left[i \frac{g_{A}^{2} m_{A}}{2 \Delta_{A}} t_{A}\right] \cos \left(\frac{g_{A}^{2} m_{A}}{4 \Delta_{A}} t_{A}\right), \\
c_{m_{A}, m_{B}}^{b, p_{-2}}\left(t_{A}\right)=i c_{m_{A}, m_{B}} \exp \left[i \frac{g_{A}^{2} m_{A}}{2 \Delta_{A}} t_{A}\right] \sin \left(\frac{g_{A}^{2} m_{A}}{4 \Delta_{A}} t_{A}\right) .
\end{gathered}
$$

These probability amplitudes of the atom-field interaction now serve as the initial conditions for the interaction of the atoms with the cavity $B$. Again the evolution of the system is similar to that with the first cavity. The final probability amplitude of the atom-field state vector after the interaction with the cavity $B$ can be obtained by using the initial conditions (5) and (6), yielding

$$
\begin{aligned}
c_{m_{A}, m_{B}}^{b, p_{0}}\left(t_{A}, t_{B}\right)= & c_{m_{A}, m_{B}} \exp \left[i \frac{g_{A}^{2} m_{A}}{2 \Delta_{A}} t_{A}+i \frac{g_{B}^{2} m_{B}}{2 \Delta_{B}} t_{B}\right] \\
& \times \cos \left(\frac{g_{A}^{2} m_{A}}{4 \Delta_{A}} t_{A}+\frac{g_{B}^{2} m_{B}}{4 \Delta_{B}} t_{B}\right), \\
c_{m_{A}, m_{B}}^{b, p_{-2}}\left(t_{A}, t_{B}\right)= & i c_{m_{A}, m_{B}} \exp \left[i \frac{g_{A}^{2} m_{A}}{2 \Delta_{A}} t_{A}+i \frac{g_{B}^{2} m_{B}}{2 \Delta_{B}} t_{B}\right] \\
& \times \sin \left(\frac{g_{A}^{2} m_{A}}{4 \Delta_{A}} t_{A}+\frac{g_{B}^{2} m_{B}}{4 \Delta_{B}} t_{B}\right),
\end{aligned}
$$

where $t_{B}$ is the interaction time of the atom with the cavity $B$. These probability amplitudes of the atom in the momentum states $\left|p_{0}\right\rangle$ and $\left|p_{-2}\right\rangle$ clearly display oscillations. The information of the field photon numbers in two cavities is contained in the oscillatory functions. Thus knowing the probabilities of the momentum states of diffracted atoms by the repeated measurements we can determine the joint photon statistics of the field state.

The final state vector of the system after the passage of the atom through the two cavities can be written as

$$
\begin{aligned}
\left|\Psi_{A, B}^{b, p_{l}}\left(t_{A}, t_{B}\right)\right\rangle= & \sum_{m_{A}, m_{B}}\left[c_{m_{A}, m_{B}}^{b, p_{0}}\left(t_{A}, t_{B}\right)\left|b, p_{0}, m_{A}, m_{B}\right\rangle\right. \\
& \left.+c_{m_{A}, m_{B}}^{b, p_{-2}}\left(t_{A}, t_{B}\right)\left|b, p_{-2}, m_{A}, m_{B}\right\rangle\right] .
\end{aligned}
$$

Detection of the atom say in momentum state $\left|p_{0}\right\rangle$ project the field state into

$$
\left\langle p_{0} \mid \Psi_{A, B}^{b, p_{l}}\left(t_{A}, t_{B}\right)\right\rangle=\frac{1}{N_{m_{A}, m_{B}}} \sum_{m_{A}, m_{B}}^{b, p_{0}}\left(t_{A}, t_{B}\right)\left|b, m_{A}, m_{B}\right\rangle,
$$

where $N$ is the normalization constant given by

$$
N=\sqrt{\sum_{m_{A}, m_{B}}\left|c_{m_{A}, m_{B}}^{b, p_{0}}\left(t_{A}, t_{B}\right)\right|^{2}} .
$$

The probability of finding the joint photon distribution after the detection of atom in momentum state $\left|p_{0}\right\rangle$ is denoted as $\mathcal{P}\left(m_{A}, m_{B}\right)$ and is given by

$$
\mathcal{P}\left(m_{A}, m_{B}\right)=\frac{1}{N^{2}} \mid c_{m_{A}, m_{B}}{ }^{2} \cos ^{2}\left[\frac{g_{A}^{2} m_{A}}{4 \Delta_{A}} t_{A}+\frac{g_{B}^{2} m_{B}}{4 \Delta_{B}} t_{B}\right] .
$$

Similarly if the atom, after interacting with both the cavities, is detected in momentum state $\left|p_{-2}\right\rangle$, then

$$
\mathcal{P}^{\prime}\left(m_{A}, m_{B}\right)=\frac{1}{N^{\prime}}\left|c_{m_{A}, m_{B}}\right|^{2} \sin ^{2}\left[\frac{g_{A}^{2} m_{A}}{4 \Delta_{A}} t_{A}+\frac{g_{B}^{2} m_{B}}{4 \Delta_{B}} t_{B}\right],
$$

where $N^{\prime}$ is given by

$$
\dot{N}=\sqrt{\sum_{m_{A}, m_{B}}\left|c_{m_{A}, m_{B}}^{b, p_{-2}}\left(t_{A}, t_{B}\right)\right|^{2}} .
$$

Thus the detection of the atoms in momentum states $\left|p_{0}\right\rangle$ or $\left|p_{-2}\right\rangle$ gives the joint photon statistics of the entangled field state, which is the product of the original joint photon statistics and a oscillatory function. If the atom is detected in momentum state $\left|p_{0}\right\rangle$, then the original joint photon statistics gets multiplied with the function $\cos ^{2}\left[g_{A}^{2} m_{A} t_{A} / 4 \Delta_{A}\right.$ $\left.+g_{B}^{2} m_{B} t_{B} / 4 \Delta_{B}\right]$. Similarly if the atom is detected in momentum state $\left|p_{-2}\right\rangle$, then the original joint photon statistics gets multiplied with the function $\sin ^{2}\left[g_{A}^{2} m_{A} t_{A} / 4 \Delta_{A}\right.$ $\left.+g_{B}^{2} m_{B} t_{B} / 4 \Delta_{B}\right]$. This function which we name the decimation function depends on $g_{i}^{2} / 4 \Delta_{i}(i=A$ or $B)$ and the interaction time between the atom and the field. The mapping of decimation function over the joint photon statistics of the entangled cavity field decimates it at the points where the product of the two functions is close to zero. This causes a reduced joint photon statistics in the cavities. It is a photon statistics of the entangled field state in two cavities which may have probabilities of the some of its joint photon number decimated to zero and also the rest of the probabilities of the set of joint photon numbers modified as the result of the multiplication with the decimation function. For the proposed scheme the quantity $g_{i}^{2} / 4 \Delta_{i}$ is constant so the spacing of the minimas of the decimation function depends upon the interaction time only. For a given interaction time the decimation functions along with the original joint photon statistics and the reduced joint photon statistics are shown in Fig. 2. Here the Fig. 2(a) is the original joint photon statistics 


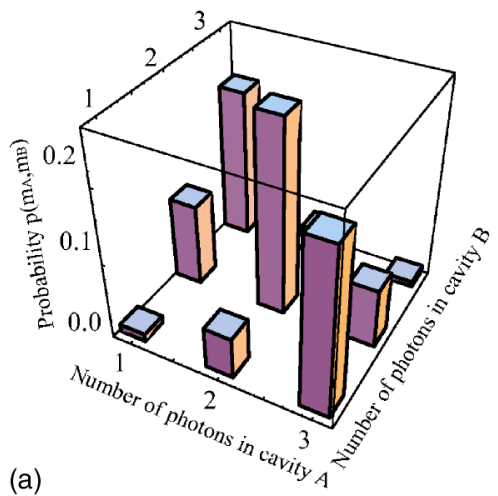

(b)
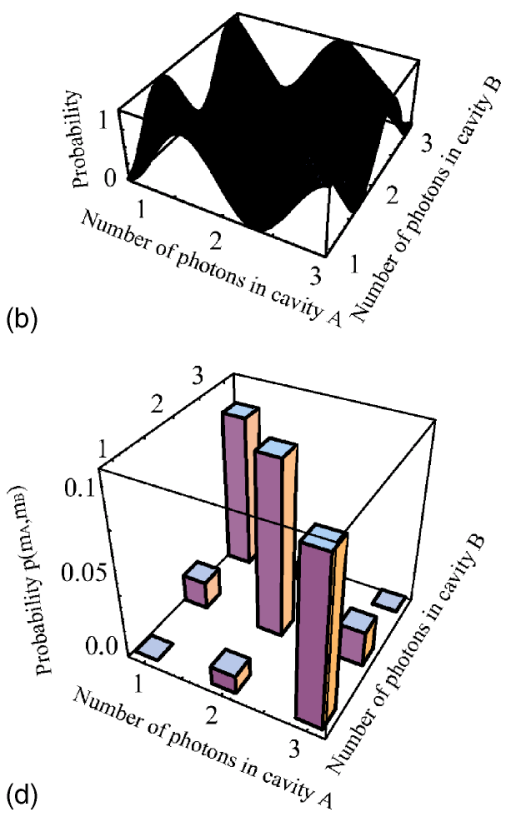

(c)

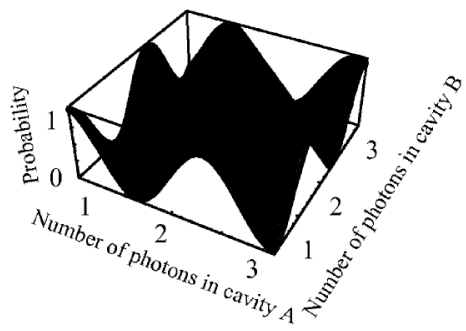

(e)

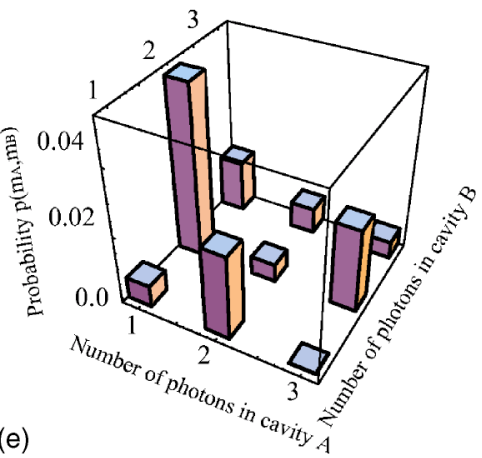

FIG. 2. (a) The original joint photon statistics of the entangled field state in two cavities. (b) The decimation function when the atom is detected in momentum $\left|p_{0}\right\rangle$. (c) The decimation function when the atom is detected in momentum $\left|p_{-2}\right\rangle$. (d) The reduced joint photon statistics corresponding to the atomic detection in momentum state $\left|p_{0}\right\rangle$. (e) The reduced joint photon statistics when the atom is detected in momentum state $\left|p_{-2}\right\rangle$. which we want to reconstruct. The detection of the atom in momentum state $\left|p_{0}\right\rangle$ or $\left|p_{-2}\right\rangle$ correspond to the decimation functions, which are plotted in Figs. 2(b) and 2(c), respectively. These two figures show the oscillatory behavior of the decimation function along increasing $m_{A}$ and $m_{B}$. The result of the mapping of the decimation function over the original joint photon statistics is shown in Figs. 2(d) and 2(e). If the atom is detected in momentum state $\left|p_{0}\right\rangle$ then the Fig. 2(d) will be the resultant reduced joint photon statistics. Alternatively if the atom is detected in momentum state $\left|p_{-2}\right\rangle$ then Fig. 2(e) will be the resultant reduced joint photon statistics. If the process is repeated with an atomic beam having velocity spread and the atoms are detected in any of the momentum state then the joint photon number distribution is suppressed and finally we arrive at fixed set of photon numbers in two cavities. Figure 3 shows the process of collapsing of the joint photon distribution to a set of joint photon number after the interaction of the 3, 6, 10 and 15 atoms in 3(a)-3(d), respectively. This situation is the extension of the schemes discussed in Ref. [8], where the field initially in a coherent state transforms to a Fock state with the measuring sequence. In our case the information acquired by detecting a sequence of atoms also modifies the entangled field function step by step, until it eventually collapses into a set of joint photon numbers. This reduced single set of joint photon numbers in the cavities can be measured by passing the atoms having fixed interaction time. This causes the atoms to be deflected after $l$ interaction in such a way that the angle of deflection of all the atoms remains the same [11]. We record the set of photon numbers and repeat the same process with an identically prepared system until we get another set of joint photon number in the two cavities. We continue the process and get the frequency of each set of photon numbers. The plot of this frequency is the reconstructed joint photon statistics shown in Fig. 4. Note that in this scheme the spread in the vertical velocity of the atom does not require any control. On the contrary, the dispersion in the atomic vertical velocity, which correspond to the uncertainty in phase helps as it makes easy to determine the entangled state of the field.

\section{WIGNER FUNCTION OF TWO-MODE ENTANGLED FIELD STATE}

The joint photon statistics alone cannot give the full information of the field. To get the information about the offdiagonal matrix elements we propose to reconstruct the 

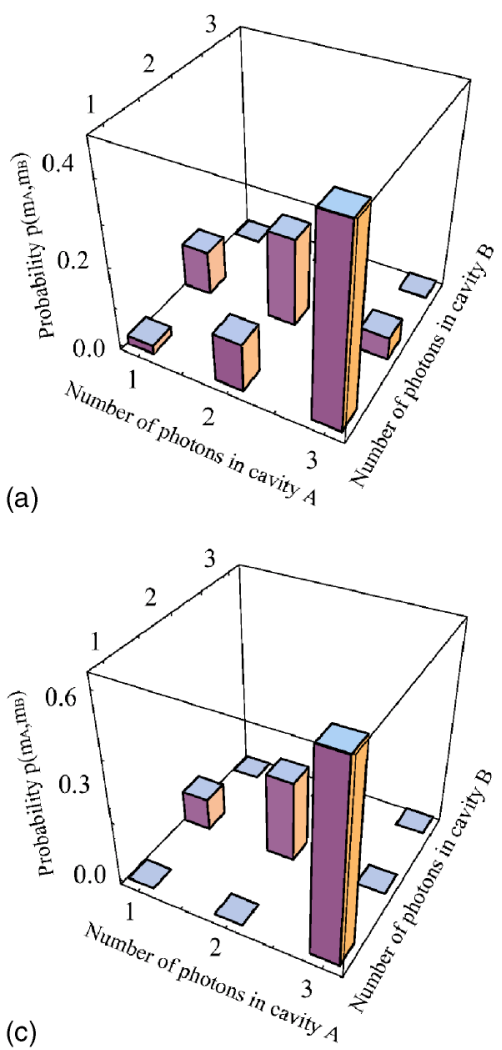
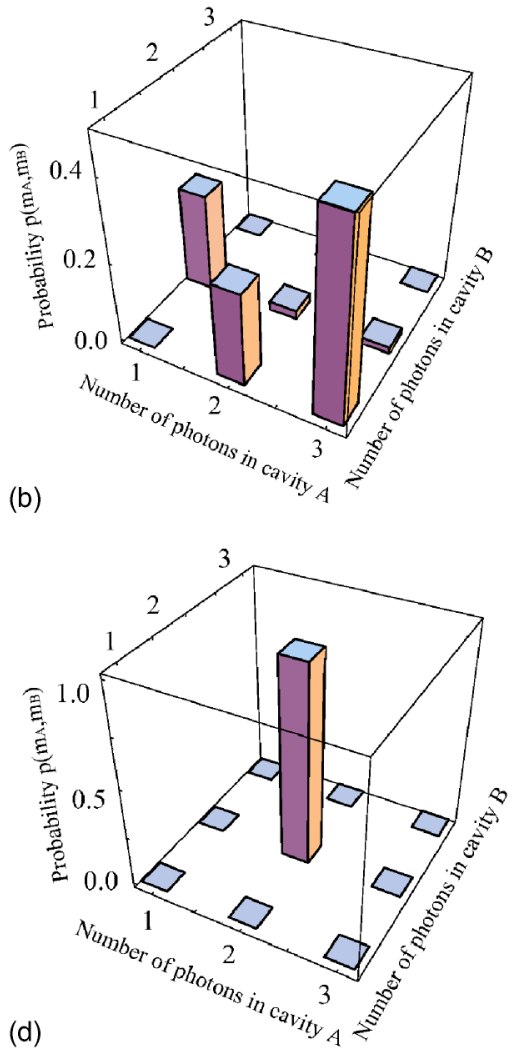

FIG. 3. Reduction of the joint photon statistics of an entangled field state $\sum_{m_{A}, m_{B}=1}^{3} c_{m_{A}, m_{B}}\left|m_{A}, m_{B}\right\rangle$ to a single set of photon numbers in two cavities. The reduced joint photon statistics after the passage of $3,6,10$, and 15 atoms are shown in (a), (b), (c), and (d), respectively.
Wigner function of the entangled field state in two separate cavities. We start with the definition of the Wigner function described in [27]. In this reference Cahill and Glauber have suggested that the Wigner function of the single mode field can be found by the measurement of the displaced cavity field state.

We extend the idea of Cahill and Glauber to a two-mode entangled field state in two separate cavities. Here we assume that there is a joint probability $p\left(m_{A}, m_{B}\right)$ of $m_{A}$ photons in cavity mode $A$ and $m_{B}$ photons in cavity mode $B$. As

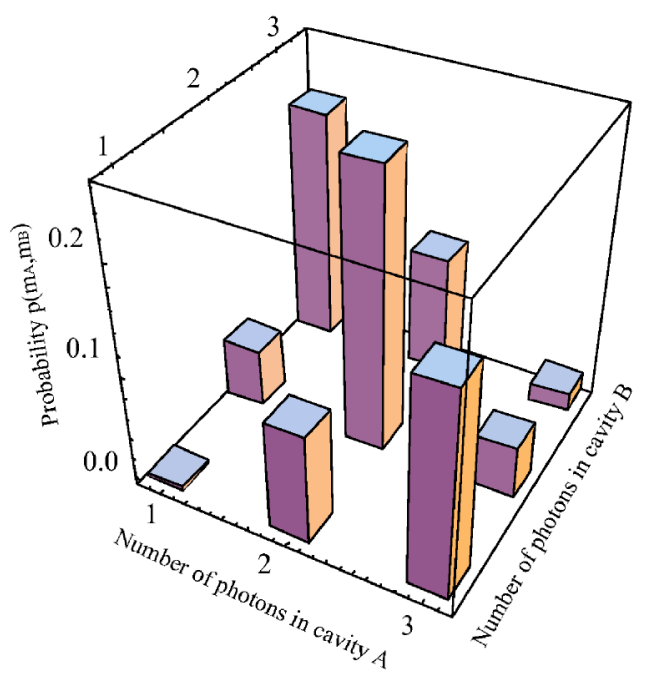

FIG. 4. Reconstructed joint photon statistics of the entangled field state in two separate cavities. During the plating of these histogram we do the simulation of 500 identical system. we want to measure the two-mode entangled field state, we displace each mode by displacement operators $D(\alpha)$ $=\exp \left[\alpha a_{A}^{\dagger}-\alpha^{*} a_{A}\right]$, and $D(\beta)=\exp \left[\beta a_{B}^{\dagger}-\beta * b_{B}\right]$, where $a_{A}\left(a_{A}^{\dagger}\right)$ and $a_{B}\left(a_{B}^{\dagger}\right)$ are the annihilation(creation) operators for the mode of electromagnetic field present in cavities $A$ and $B$, respectively. The quasiprobability distribution corresponding to the two-mode entangled field states is defined as

$$
\Theta\left(\alpha, \alpha^{*}, \beta, \beta *\right)=\frac{1}{\pi^{2}} \operatorname{Tr}[\rho T(\alpha, \beta, s)],
$$

where $s$ yields the order of products of the field operators and $\alpha, \beta$ are the injected coherent states. The term $T(\alpha, \beta, s)$ defined in Ref. [26] is the two dimensional Fourier transform of the displacement operators $D(\alpha)$ and $D(\beta)$.

For $s=0$ we obtain the Wigner distribution and for $s=-1$, and $1, Q$ and $P$ representations, respectively. The quasiprobability distribution function defined in Eq. (13) gets the form of Wigner function for a general state $\rho=|\Psi\rangle\langle\Psi|$, as

$$
W(\alpha, \alpha *, \beta, \beta *)=\frac{4}{\pi^{2}} \sum_{m_{A}, m_{B}}(-1)^{m_{A}+m_{B}} p\left(m_{A}, m_{B}, \alpha, \beta\right)
$$

where $p\left(m_{A}, m_{B}, \alpha, \beta\right)$ is the joint photon statistics of the displaced entangled cavities field, i.e.,

$$
p\left(m_{A}, m_{B}, \alpha, \beta\right)=\left|\left\langle m_{A}, m_{B}\left|D^{\dagger}(\beta) D^{\dagger}(\alpha)\right| \Psi\right\rangle\right|^{2} .
$$

Thus the Wigner function of the field can be found directly if the displaced photon statistics $p\left(m_{A}, m_{B}, \alpha, \beta\right)$ is known for all values of $\alpha$ and $\beta$. 
In the previous section we have presented a scheme for the measurement of the photon statistics of the undisplaced entangled field state in two cavities. To get the Wigner function of the entangled state we propose to displace each mode by injecting coherent states $|\alpha\rangle$ and $|\beta\rangle$ into the cavities $A$ and $B$, respectively. Experimentally this operation can be carried out by coupling two resonant classical oscillators to the cavities $A$ and $B$, respectively. The joint photon statistics of the displaced entangled field can be obtained by the procedure mentioned earlier, i.e., by passing two-level offresonant atoms from the two cavities and then measuring its momentum state. The measuring sequence gives the photon statistics of the displaced entangled state. By repeating the measurement of identically prepared systems but with different values of $\alpha$ and $\beta$, we can reconstruct the Wigner function $W\left(\alpha, \alpha^{*}, \beta, \beta^{*}\right)$ in a straight forward way as shown in Eq. (14).

\section{RESULT AND DISCUSSION}

We have presented a scheme to reconstruct the Wigner function of an entangled field state in two separate cavities. In the first step we presented a scheme for the measurement of joint photon statistics of the entangled field. For this purpose we use atomic Bragg diffraction of two-level atoms from the entangled field in the cavities. In this scheme we consider the large detuning between the entangled field frequencies and the atomic transition frequency. This ensures that the atoms do not exit from the cavity in excited state and there is no spontaneous emission. The information of the joint field photon numbers can be extracted from the momentum probability distribution of the deflected atoms. We see from our calculations that the momentum probability of the diffracted atoms is a product of initial photon statistics and a decimation function whose argument contains the information of the field photon numbers. In order to find the joint photon statistics of the entangled field state, we use the method of reducing it to a single set of joint photon number state and then to measure it. Interaction of each atom with the entangled field present in two separate cavities updates the cavities field statistics depending upon the interaction time. The joint photon statistics gets multiplied by the so called decimation function, which has periodic maximas and minimas. The position of the minimas changes with the interaction time of the atom with the field. Each atom in different interaction time eliminates some photon numbers probabilities in the field distribution. After the passage of a few atoms from the cavities only one set of photon number state is left, which does not change by further interaction of the atoms with field. We display this procedure in Fig. 3. By repeating this process on identically prepared systems and getting the frequency distribution of each set of photon numbers we can reconstruct the photon distribution in two cavities. Figure 4 illustrates the photon statistics which is reproduced on the basis of proportion of times each joint photon state is selected. A comparison of original distribution with the reconstructed one displays a good agreement.

The Bragg's diffraction of the atomic de-Broglie wave can be realized, for example, as discussed in Ref. [30]. In this paper Dürr and Rempe report the Bragg's diffraction of ${ }^{85} \mathrm{Rb}$ atoms with an optical field of wavelength $\lambda=800 \mathrm{~nm}$. In this case the recoil frequency is $\hbar k^{2} / 2 M \cong 23.05 \mathrm{KHz}$, the detuning is $\Delta=502.65 \mathrm{MHz}$ and the coupling constant of the atom field system is $g=703.72 \mathrm{KHz}$. For realizing Bragg's diffraction of ${ }^{85} \mathrm{Rb}$ atoms one needs cold atoms of temperature range $10^{-2} \mathrm{~K}$. The cavities life time should be large enough as compared to the total interaction time of the atoms with the cavity.

Our scheme consists of two steps: In first step, we send the atoms with a velocity spread and detect them in momentum $P_{0}$ and $P_{-2}$. It reduces the field in the cavities, until we get a single set of joint photon number in two cavities. Our simulation shows that around 15 atoms are enough for this reduction. After the reduction of the photon distribution to a set of joint photon number, the additional atoms send will not alter the joint photon number because the reduced state of the field is a function of interaction time only (as the of joint photon number has be suppressed). In the second step, we measure the reduced state by sending fixed velocity atoms and detecting them in at a particular momentum state, i.e., $P_{0}$ or $P_{-2}$. If we select the detection of atoms in momentum state $P_{-2}$ then in this case, for a reduced set of joint photon number $\left(m_{A}, m_{B}\right)$ the velocity selection (this corresponds to the interaction time of the atoms) should be such that the argument of the cosine or sine function must be $\pi / 2$. This gives us the probability of the momentum state $P_{-2}$ equal to 1. Around 5 atoms are sufficient for this measurement. In average we need 20 atoms for whole process. As mentioned earlier the cavity life time should be larger than the total interaction time of the atoms with the cavities. Using very high finesse cavities with high- $Q$ factors may realize this scheme. We can realize this scheme in optical regime using lighter atom such as $\mathrm{He}$, which has a mass of 6.64 $\times 10^{-27} \mathrm{Kg}$ and emission line at wave length $\lambda=543.5 \mathrm{~nm}$. One can choose temperature $230 \mathrm{~K}$. The recoil energy is $\hbar k^{2} / 2 M \cong 1.06 \mathrm{MHz}$. For detuning $6.28 \mathrm{GHz}$ the effective Rabi frequency is $g^{2} \sqrt{m} / 4 \Delta \cong 208 \mathrm{KHz}$ (for maximum of $m=3$ photons in mode $A$ ). The interaction time with one cavity is approximately $8.3 \mu \mathrm{s}$. The total interaction time for 20 atoms comes as $166 \mu \mathrm{s}$. As the atom passes through the two cavities so one may approximate that the interaction time will be the double of the one cavity total interaction time. Moreover, as there will be some distance between the two cavities, we suggest to take a separation of $1 \mathrm{~cm}$ between the cavities. As the cavity width is taken $1 \mathrm{~cm}$ in the simulation so the total time required will be three times of $166 \mu$ s (i.e., $498 \mu$ s). For this required lifetime of the cavities one may use the cavities discussed by the Kimble et al. group [31]. The finesse $\mathcal{F}=7.85 \times 10^{6}$ could be used in such a way that the spacing between the mirrors should be $30 \mathrm{~cm}$. This corresponds to a lifetime of $2.5 \mathrm{~ms}$ of the cavities (as the finesse is related with the lifetime of the cavity via $t_{c}$ $=\mathcal{F} d / \pi c$, where $c$ is the velocity of the light and $d$ is the spacing of the cavity mirrors). This cavity lifetime is reasonable to realize our scheme.

The joint photon statistics of the cavity field allows us to calculate the Wigner function. The complete Wigner function can be obtained by shifting the system or equivalently the 
frame of reference in phase space. This can be obtained by attaching coherent states with the signal field. We displace each mode of the entangled field by injecting coherent states $|\alpha\rangle$ and $|\beta\rangle$ resonant to the field modes $A$ and $B$, respectively. The measurement of the displaced photon statistics gives the complete Wigner function in a straightforward manner.

\section{ACKNOWLEDGMENTS}

The research of M.S.Z. is supported by the Air Force Office of Scientific Research, Air Force Research Laboratory (Rome, NY), TAMU Telecommunication and Informatics Task Force (TITF) initiative, and DARPA (QuIST).
[1] P. W. Shor, SIAM J. Comput. 26, 1484 (1997); L. K. Grover, Phys. Rev. Lett. 79, 325 (1997); 79, 4709 (1997).

[2] C. H. Bennett, G. Brassard, C. Crepeau, R. Jozsa, A. Peres, and W. K. Wootters, Phys. Rev. Lett. 70, 1895 (1993); M. Ikram, S. Y. Zhu, and M. S. Zubairy, Phys. Rev. A 62, 022307 (2000).

[3] A. K. Ekert, Phys. Rev. Lett. 67, 661 (1991); A. K. Ekert, J. G. Rarity, P. R. Tapster, and G. MassimoPalma, ibid. 69, 1293 (1992).

[4] C. H. Bennett and S. J. Wiesner, Phys. Rev. Lett. 69, 2881 (1992).

[5] S. F. Huelga, C. Macchiavello, T. Pellizzari, A. K. Ekert, M. B. Plenio, and J. I. Cirac, Phys. Rev. Lett. 79, 3865 (1997); S. Bose, V. Vedral, and P. L. Knight, Phys. Rev. A 57, 822 (1998); M. Murao, M. B. Plenio, S. Popescu, V. Vedral, and P. L. Knight, ibid. 57, R4075 (1998); A. Karlsson, M. Koashi, and N. Imoto, ibid. 59, 162 (1999); P. W. Shor, ibid. 52, R2493 (1995).

[6] D. T. Smithey, M. Beck, M. G. Raymer, and A. Faridani, Phys. Rev. Lett. 70, 1244 (1993); S. Schiller, G. Breitenbach, S. F. Pereira, T. Muller, and J. Mlynek, ibid. 77, 2933 (1996).

[7] K. Vogel and H. Risken, Phys. Rev. A 40, 2847 (1989).

[8] M. Brune, S. Haroche, V. Lefevre, J. M. Raimond, and N. Zagury, Phys. Rev. Lett. 65, 976 (1990); M. Brune, S. Haroche, J. M. Raimond, L. Davidovich, and N. Zagury, Phys. Rev. A 45, 5193 (1992).

[9] M. S. Zubairy, Phys. Lett. A 222, 91 (1996); P. J. Bardroff, E. Mayr, and W. P. Schleich, Phys. Rev. A 51, 4963 (1995); M. S. Zubairy, ibid. 57, 2066 (1998); T. Azim and M. S. Zubairy, Phys. Lett. A 250, 344 (1998); M. Mahmoudi, H. Tajalli, and M. S. Zubairy, J. Opt. B: Quantum Semiclassical Opt. 2, 315 (2000)

[10] M. Freyberger and A. M. Herkommer, Phys. Rev. Lett. 72, 1952 (1994).

[11] A. A. Khan and M. S. Zubairy, Phys. Lett. A 254, 301 (1999).

[12] Ashfaq H. Khosa and M. S. Zubairy (unpublished).

[13] A. F. Bernhardt and B. W. Shore, Phys. Rev. A 23, 1290 (1981)

[14] P. Meystre, E. Schumacher, and S. Stenholm, Opt. Commun. 73, 443 (1989).
[15] M. J. Holland, D. F. Walls, and P. Zoller, Phys. Rev. Lett. 67, 1716 (1991); A. M. Herkommer, V. M. Akulin, and W. P. Schleich, ibid. 69, 3298 (1992).

[16] M. Marte and S. Stenholm, Appl. Phys. B: Photophys. Laser Chem. 54, 443 (1992).

[17] P. L. Gould, P. J. Martin, G. A. Ruff, R. E. Stoner, J. L. Picque, and D. E. Pritchard, Phys. Rev. A 43, 585 (1991), J. Lawall and M. Prentiss, Phys. Rev. Lett. 72, 993 (1994).

[18] L. S. Goldner, C. Gerz, R. J. C. Spreeuw, S. L. Rolston, C. I. Westbrook, W. D. Phillips, P. Marte, and P. Zoller, Phys. Rev. Lett. 72, 997 (1994); D. M. Giltner, R. W. McGowan, and Siu Au Lee, Phys. Rev. A 52, 3966 (1995).

[19] C. J. Hood, M. S. Chapman, T. W. Lynn, and H. J. Kimble, Phys. Rev. Lett. 80, 4157 (1998).

[20] M. G. Raymer, D. F. McAlister, and U. Leonhardt, Phys. Rev. A 54, 2397 (1996).

[21] M. S. Kim and G. S. Agarwal, Phys. Rev. A 59, 3044 (1999).

[22] H. Kuhn, D. G. Welsch, and W. Vogel, Phys. Rev. A 51, 4240 (1995).

[23] M. Ikram and M. S. Zubairy, Phys. Rev. A 65, 044305 (2002).

[24] M França Santos, L. G. Lutterbach, and L. Davidovich, J. Opt. B: Quantum Semiclassical Opt. 3, 15214 (2001).

[25] M. S. Kim, G. Antesberger, C. T. Bodendorf, and H. Walther, Phys. Rev. A 58, R65 (1998); M. Wilkens and P. Meystre, ibid. 43, 3832 (1991); S. M. Dutra, P. L. Knight, and H. MoyaCessa, ibid. 48, 3168 (1993).

[26] L. G. Lutterbach and L. Davidovich, Phys. Rev. Lett. 78, 2547 (1997).

[27] K. E. Cahill and R. J. Glauber, Phys. Rev. 177, 1857 (1969); 177, 1882 (1969).

[28] K. Banaszek and K. Wodkiewicz, Phys. Rev. Lett. 76, 4344 (1996).

[29] L. Davidovich, N. Zagury, M. Brune, J. M. Raimond, and S. Haroche, Phys. Rev. A 50, R895 (1994); J. A. Bergou, J. Mod. Opt. 44, 1957 (1997); M. Ikram, S. Y. Zhu, and M. S. Zubairy, Opt. Commun. 184, 417 (2000).

[30] S. Dürr and G. Rempe, Phys. Rev. A 59, 1495 (1999).

[31] Christina J. Hood, H. J. Kimble, and Jun Ye, Phys. Rev. A 64, 033804 (2001). 